\title{
Acoustic emission monitoring of medieval towers considered as sensitive earthquake receptors
}

\author{
A. Carpinteri, G. Lacidogna, and G. Niccolini \\ Politecnico di Torino, Department of Structural Engineering \& Geotechnics, Turin, Italy
}

Received: 20 November 2006 - Accepted: 14 March 2007 - Published: 2 April 2007

\begin{abstract}
Many ancient masonry towers are present in Italian territory. In some cases these structures are at risk on account of the intensity of the stresses they are subjected to due to the high level of regional seismicity. In order to preserve this inestimable cultural heritage, a sound safety assessment should take into account the evolution of damage phenomena. In this connection, acoustic emission (AE) monitoring can be highly effective.

This study concerns the structural stability of three medieval towers rising in the centre of Alba, a characteristic town in Piedmont (Italy). During the monitoring period a correlation between peaks of AE activity in the masonry of these towers and regional seismicity was found. Earthquakes always affect structural stability. Besides that, the towers behaved as sensitive earthquake receptors. Here a method to correlate bursts of AE activity in a masonry building and regional seismicity is proposed. In particular, this method permits to identify the premonitory signals that precede a catastrophic event on a structure, since, in most cases, these warning signs can be captured well in advance.
\end{abstract}

\section{Introduction}

Non-destructive and instrumental investigation methods are currently employed to measure and check the evolution of adverse structural phenomena, such as damage and cracking, and to predict their subsequent developments. In particular, damage assessment for historical masonry buildings is often a complex task (Carpinteri and Bocca, 1991; Anzani et al., 2000). It is essential to distinguish between stable damage patterns and damage evolution leading to a catastrophic structural collapse. Some damage patterns can be subsequently activated by unpredictable events such as

Correspondence to: A. Carpinteri

(alberto.carpinteri@polito.it) earthquakes, or by inappropriate functional extensions and restorations. In addition, the limited ductility of the masonry, combined with the large scale of these buildings, provides a rather brittle structural behaviour (Carpinteri et al., 2005). Damage, cracking and the evolution of these phenomena over time taking place in some portions of the masonry structures can be assessed through the Acoustic Emission (AE) technique (Carpinteri et al., 2007)

This study concerns the structural stability of three medieval towers, "Torre Sineo", "Torre Astesiano" and "Torre Bonino" (Fig. 1) rising in the centre of Alba, a characteristic town in Piedmont (Italy) (Carpinteri and Lacidogna, 2006b). During the monitoring period a correlation between peaks of $\mathrm{AE}$ activity in the masonry of these towers and regional seismicity is found. In fact the towers behaved as sensitive earthquake receptors.

Earthquake statistics exhibit a complicated spatiotemporal behaviour which reflects the extreme complexity of Earth's crust. Despite this complexity, there is a universally valid scaling law: the frequency-magnitude statistics of earthquakes given by the Gutenberg-Richter (GR) relation (Richter, 1958). On the other hand, AE in materials and earthquakes in Earth's crust, though they take place on very different scales, are very similar and correlated in time. In either case there is a release of elastic energy from localised sources inside a medium: respectively opening microcracks and hypocentres of earthquakes. Furthermore, earthquakes always affect structural stability. This similarity suggests an interpretation in which $\mathrm{AE}$ and seismic events are now considered to be linked both in space and time. With this approach it is possible to find correlation between bursts of $\mathrm{AE}$ activity in a masonry building and regional seismicity. In conclusion, this approach can be used to identify the premonitory signals that precede a catastrophic event on a structure, since, in most cases, these warning signs can be captured well in advance.

Published by Copernicus GmbH on behalf of the European Geosciences Union. 


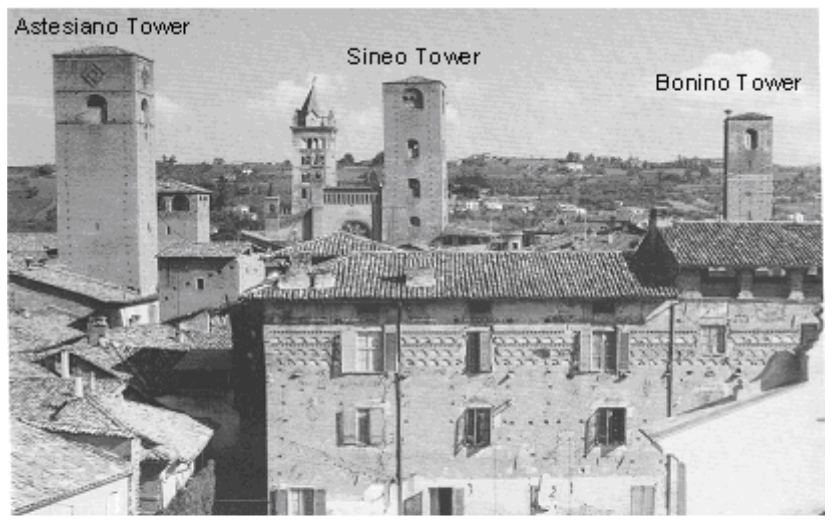

Fig. 1. An overview of the medieval towers of Alba.

\section{Description of the three towers}

These masonry buildings from the 13th Century are the tallest and mightiest medieval towers preserved in Alba (Fig. 1).

The Sineo Tower is square-based, $39 \mathrm{~m}$ high, and leans to one side by about $1 \%$. Wall thickness ranges from $2 \mathrm{~m}$ at the foundation level to $0.8 \mathrm{~m}$ at the top. The bearing walls are a sacco, i.e., consist of brick faces enclosing a mixture of rubble and bricks bonded with lime and mortar. Over a height of $15 \mathrm{~m}$, the tower is incorporated in a later building. The Astesiano Tower has a similar structure, but has a rectangular base. The filling material is more organised, with brick courses arranged in an almost regular fashion, which, however, are not connected with the outer wall faces. In this case too, the total thickness of the masonry ranges from $2 \mathrm{~m}$ at the bottom to $0.8 \mathrm{~m}$ at the top. Total height is ca $36 \mathrm{~m}$ and the tower does not lean on any side. It is also incorporated in a later building, approximately $15 \mathrm{~m}$ high, built when the tower had been completed. The Bonino Tower, just under $35 \mathrm{~m}$ high, is the least imposing of the towers analysed. The bearing walls are similar to those of the Astesiano Tower, except that the lower storeys are coated with a thick layer of plaster of over $10 \mathrm{~cm}$. The thickness of the walls ranges from $1.8 \mathrm{~m}$ at the base to $0.6 \mathrm{~m}$ at the top. The square-based structure has been incorporated in a valuable building from the Italian Art Nouveau period.

During the monitoring of AE activity in the masonry of these towers regional seismic activity occurred (Carpinteri and Lacidogna, 2006b). AE activity on the Sineo Tower, recorded in several monitoring time windows, and earthquakes in local Richter magnitude $M_{L}$ scale occurred in the Alba region during the whole AE monitoring period are reported in Table 1. The earthquakes with their parameters including epicentral position and hypocentral depth are listed in Table 2. The earthquake parameters have been provided by the National Institute for Geophysics and Volcanology in Rome.

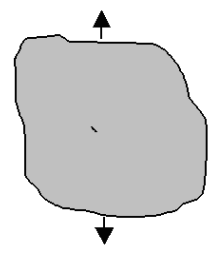

(a)

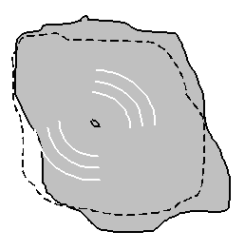

(b)

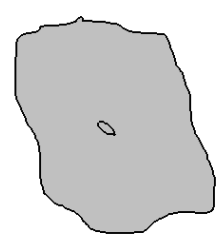

(c)
Fig. 2. Acoustic Emission in a structural element subjected to deformation during damage process. Early stage of the initiating microcrack (a). Opening of the microcrack (b). Arrest of the microcrack (c).

\section{Damage detection of the towers}

\subsection{Acoustic emission equipment and signals analysis}

The cracking processes taking place in some portions of the masonry structures were then monitored using the AE technique. Crack opening, in fact, is accompanied by the emission of elastic waves which propagate within the bulk of the material. In general, a microcrack is nucleated by the rupture of a bond in a weak spot, see Fig. 2a. The stress on the failed bond is suddenly redistributed to the remaining sound bonds by waves which propagate through the elastic material, see Fig. 2b. The transient stress wave ends when a new equilibrium configuration is reached, see Fig. 2c.

Acoustic emission (AE) is referred to as the release of elastic waves due to microcracking. These waves can be captured and recorded by transducers applied to the surface of the structural elements (Carpinteri et al., 2004, 2007). The $\mathrm{AE}$ measurement system Atel ${ }^{\circledR}$ used by the authors consists of eight piezoelectric (PZT) transducers, calibrated on inclusive frequencies between 50 and $500 \mathrm{kHz}$, and eight control units. The threshold level for the signals recorded by the equipment, fixed at $100 \mu \mathrm{V}$, is amplified up to $100 \mathrm{mV}$. The system does not provide for the analysis of signal frequency. The amplification gain, given the relationship $\mathrm{dB}=20 \log _{10} \quad E \mathrm{u} / E \mathrm{i}$, where $E \mathrm{u} / E \mathrm{i}$, is the ratio between the input voltage and the output voltage, turns out to be $60 \mathrm{~dB}$. This is the signal amplification value generally adopted in monitoring AE events in masonry and in concrete. The oscillation counting limit has been fixed at 255 oscillations every $120 \mathrm{~s}$. This procedure is termed as Ring-Down counting, where the number of counts is proportional to crack advancement (Brindley, 1973; Pollock, 1973; Carpinteri and Lacidogna, 2006a). The exponential decay of this signal with respect to time, for a single cracking event, is shown in Fig. 3.

\subsection{Acoustic emission monitoring}

For the Sineo Tower, through AE monitoring, two cracks were detected in the inner masonry layer at the seventh floor level (Fig. 4). The monitoring process revealed an on-going damaging process, characterized by slow crack propagation 
Table 1. Number of earthquakes and AE paroxysm recorded on the Sineo Tower from 16 September 2000 to 7 November 2000.

\begin{tabular}{|c|c|c|c|c|c|}
\hline \multicolumn{2}{|c|}{ Time Window of AE Monitoring } & \multicolumn{2}{|c|}{ Seismic Events } & \multicolumn{2}{|c|}{ AE Counts } \\
\hline From & To & Time & $\mathbf{M}_{L}^{(1)}$ & Crack n.1 & Crack n.2 \\
\hline 16 Sep 2000 & 18 Sep 2000 & 16 Sep 2000 & 2.7 & 0 & 0 \\
\hline h 12.00 & h 16.00 & h 22.04 & & & \\
\hline 18 Sep 2000 & 22 Sep 2000 & & & 1052 & 712 \\
\hline h 17.00 & h 17.00 & & & & \\
\hline 22 Sep 2000 & 26 Sep 2000 & 26 Sep 2000 & 2.4 & 255 & 255 \\
\hline h 17.00 & h 11.00 & h 02.41 & & & \\
\hline 26 Sep 2000 & 29 Sep 2000 & & & 430 & 75 \\
\hline h 11.00 & h 11.00 & & & & \\
\hline 29 Sep 2000 & 3 Oct 2000 & & & 0 & 0 \\
\hline h 11.00 & h 11.00 & & & & \\
\hline 3 Oct 2000 & 7 Oct 2000 & 4 Oct 2000 & 3.0 & 405 & 1337 \\
\hline h 11.00 & h 11.00 & h 16.34 & & & \\
\hline 7 Oct 2000 & 13 Oct 2000 & 9 Oct 2000 & 2.5 & 310 & 233 \\
\hline h 11.00 & h 11.00 & $\begin{array}{l}\text { h } 22.43 \\
12 \text { Oct } 2000 \\
\text { h } 06.19\end{array}$ & 2.9 & & \\
\hline 13 Oct 2000 & 19 Oct 2000 & 15 Oct 2000 & 2.7 & 248 & 50 \\
\hline h 11.00 & h 11.00 & h 18.01 & & & \\
\hline 19 Oct 2000 & 24 Oct 2000 & 20 Oct 2000 & 2.8 & 262 & 336 \\
\hline h 11.00 & h 11.00 & h 01.30 & & & \\
\hline 24 Oct 2000 & 27 Oct 2000 & & & 17 & 17 \\
\hline h 11.00 & h 11.00 & & & & \\
\hline 27 Oct 2000 & 31 Oct 2000 & 27 Oct 2000 & 3.2 & 11 & 60 \\
\hline h 11.00 & h 11.00 & h 14.37 & & & \\
\hline 31 Oct 2000 & 4 Nov 2000 & & & 66 & 261 \\
\hline h 11.00 & h 11.00 & & & & \\
\hline 4 Nov 2000 & 7 Nov 2000 & & & 0 & 0 \\
\hline h 11.00 & h 11.00 & & & & \\
\hline
\end{tabular}

${ }^{(1)}$ Local Richter scale magnitude

Table 2. Earthquakes in local Richter magnitude $\mathrm{M}_{L}$ scale occurred in Alba region from 16 September 2000 to 7 November 2000.

\begin{tabular}{|c|c|c|c|c|c|c|}
\hline Hypocentral Latitude & Hypocentral Longitude & Hypocentral Depth [km] & $\begin{array}{l}\text { Epi. }^{(1)} \\
{[\mathrm{km}]}\end{array}$ & Hypo. $^{(2)}[\mathrm{km}]$ & $M_{L}^{(3)}$ & Time \\
\hline 45.078 & 8.027 & 28.728 & 42.75 & 51.50 & 2.7 & 16 Sep 2000 \\
\hline 44.785 & 8.283 & 5.000 & 22 & 22.56 & 2.4 & 26 Sep 2000 \\
\hline 44.828 & 8.330 & 5.000 & 27.5 & 27.95 & 3.0 & 4 Oct 2000 \\
\hline 44.865 & 9.376 & 10.000 & 108.75 & 109.20 & 2.5 & 9 Oct 2000 \\
\hline 44.862 & 8.339 & 5.000 & 30.5 & 30.90 & 2.9 & 12 Oct 2000 \\
\hline 44.063 & 8.584 & 10.000 & 82.25 & 82.85 & 2.7 & 15 Oct 2000 \\
\hline 44.837 & 8.341 & 5.000 & 28 & 28.44 & 2.8 & 20 Oct 2000 \\
\hline 44.829 & 8.364 & 5.000 & 30.25 & 30.66 & 3.2 & 27 Oct 2000 \\
\hline
\end{tabular}

(1) Epicentral distance from Alba (km)

(2) Hypocentral distance from Alba $(\mathrm{km})$

(3) Local Richter scale magnitude

inside the brick walls. In the most damaged zone, crack spreading had come to a halt, the cracks having achieved a new condition of stability, leading towards compressed zones of the masonry. In this particular case it can be seen that, in 


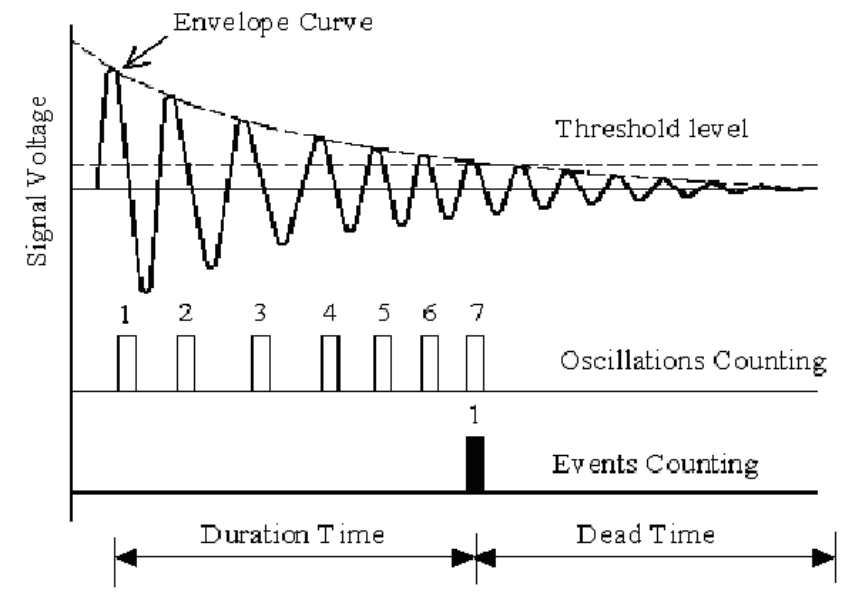

Fig. 3. Signal identified by the transducer and counting methods in AE technique.

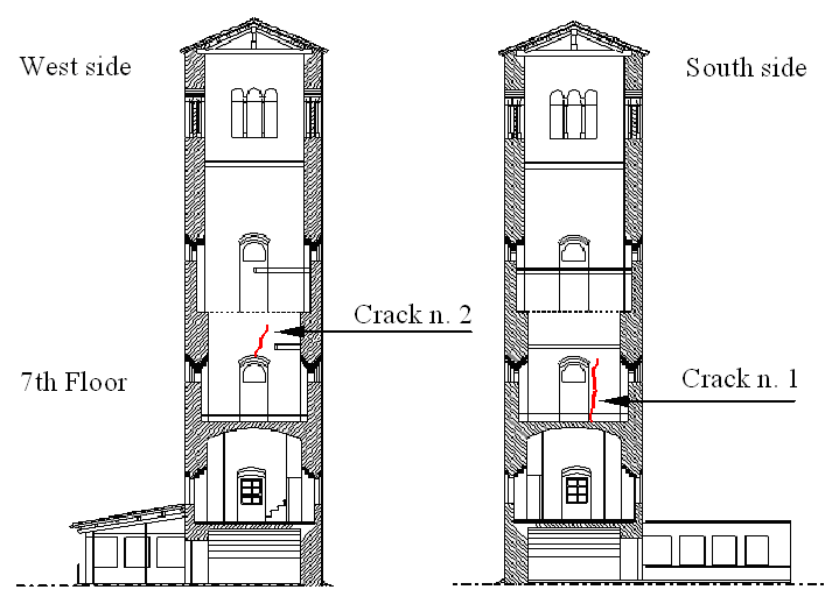

Fig. 4. Cross-sections of two sides of the Sineo Tower. Notice the presence of two cracks near the openings at the seventh floor level.

the zone monitored, each appreciable crack advance is often correlated to a seismic event. In the diagram shown in Fig. 5a, the time progression of the total ring-down count relative to AE activity of Crack no. 1 is illustrated. Furthermore, this cumulative AE function is overlapped to the seismic events recorded in the Alba region during the same time period (see Table 1 and the geographic map in Fig. 6). The relative intensity of the seismic events is also shown in Fig. 5a.

A similar behaviour was observed for the Astesiano Tower. This structure was monitored by means of two transducers applied to the inner masonry layer of the tower, at the fourth floor level near the tip of a long vertical crack. The results obtained during the monitoring period are summarised in the diagram in Fig. 5b. It can be seen how the damage to the masonry and the propagation of the crack, as reflected by the cumulative number of EA events, evolved progressively in
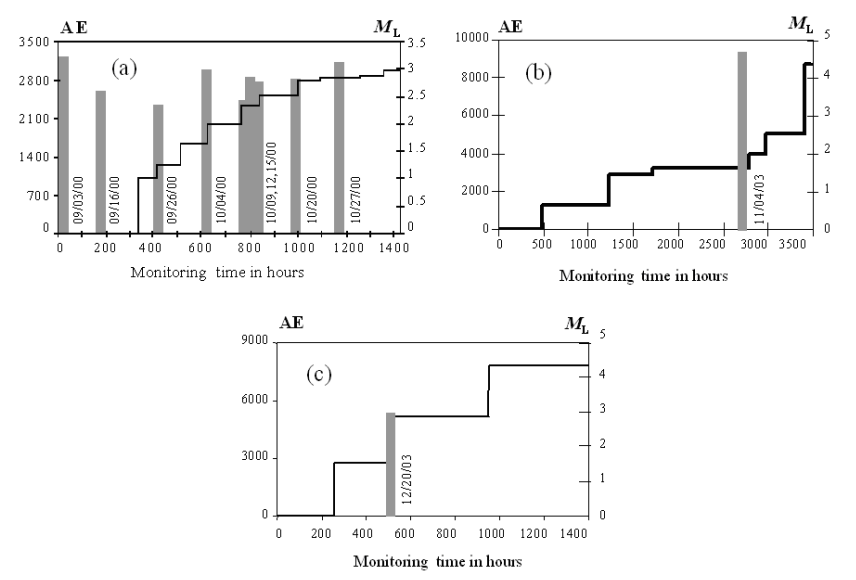

Fig. 5. AE counting number and seismic events in local Richter scale magnitude $\left(M_{L}\right)$. Sineo Tower (a), Astesiano Tower (b), Bonino Tower (c).

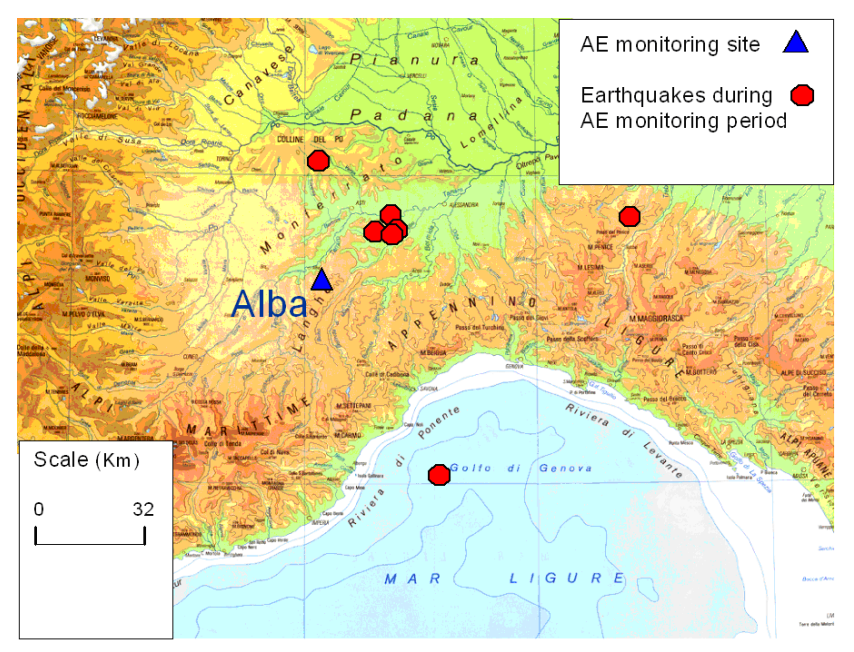

Fig. 6. Geographic map showing the AE monitoring site and the regional seismic activity occurred during the time of AE monitoring of Tower Sineo (from 16 September 2000 to 7 November 2000).

time. A seismic event of magnitude 4.7 on the Richter scale occurred during the monitoring period: from the diagram we can see how the cumulative function of EA counts grew rapidly immediately after the earthquake. The monitoring of the Bonino Tower was performed at first floor level, where the effects of restructuring works have affected the masonry most adversely. Under constant loading, a progressive release of energy is observed, due to a creep phenomenon in the material. A seismic event of magnitude 3 on the Richter scale occurred during the monitoring period (Fig. $5 \mathrm{c}$ ).

During the observation period, the towers behaved as sensitive earthquake receptors. Thus, as can be seen, the $\mathrm{AE}$ technique is able to analyse state variations in a certain physical system and can be used as a tool for predicting the occurrence of catastrophic events. In many physics problems - 
e.g., when studying test specimen failure in a laboratory, the modalities of collapse of a civil structure, the natural seismic activity of a volcano or the localisation of the epicentral volume of an earthquake - the modalities of a structural collapse are generally analysed after the event. This technique can be used instead to identify the premonitory signals that precede a catastrophic event, as, in most cases, these warning signs can be captured well in advance (Zapperi et al., 1997; Gregori and Paparo, 2004; Gregori et al., 2005).

\subsection{The "b-value" analysis}

A magnitude-frequency empirical relation, the celebrated GR relation (Richter, 1958), originally introduced by Gutenberg and Richter to describe the earthquakes statistics, has been later successfully applied in the acoustic emission field:

$N(\geq m)=10^{a-b m}$,

where $N$ is the number of $\mathrm{AE}$ events with magnitude $\geq m$ in the monitored structural element, and $b$ and $a$ are positive coefficients to be determined subjecting collected AE data to a statistical analysis. In particular, $b$ is a parameter which says how large the ratio of events with low magnitude, i.e. smaller microcracks, to large ones (that is with higher magnitude) is. From the literature on AE tests it is well-known that the $b$-value decreases as the monitored specimen approaches impending failure. It is common to observe a trend of $b$ value to the critical value $b_{\text {crit }}=1$ during final crack propagation (Colombo et al., 2003).A theoretical basis for explaining $b_{\text {crit }}=1$ has been established, by exploiting properties of a power-law crack size distribution (Carpinteri, 1994; Carpinteri et al., 2006).

Besides the released energy, through the ring-down counting it is possible to give a good estimation to the $b$-value of the GR relation (a more precise evaluation of $b$-value can be achieved with more sophisticated AE acquisition systems, see Carpinteri et al., 2006) by the formula:

$b=\frac{\log _{10} n_{\mathrm{MAX}}-\log _{10} n_{0}}{\log _{10} A_{t h}}$,

where $A_{t h}$ is the threshold voltage, $n_{\mathrm{MAX}}$ is the counting capacity of the transducers, and $n_{0}$ is the number of ring-downs counted in a specified time window. Therefore, the $b$-value given by Eq. (2) is a saturation index of the counting capability the transducers, and it depends on the adopted transducers and the fixed threshold. Low values of $b$ correspond to high values of $n_{0}$ indicating a large amount of $\mathrm{AE}$ activity, i.e. a large damage amount, whereas high values of $b$ correspond to low values of $n_{0}$ indicating a small amount of AE activity, i.e. a small damage amount.

For the towers $\mathrm{AE}$ monitoring the Atel ${ }^{\circledR}$ equipment has been adopted (Carpinteri and Lacidogna, 2006a, b), with a threshold level fixed at $A_{t h}=100 \mathrm{mV}$, and the oscillation counting limit fixed at 255 oscillations every $120 \mathrm{~s}$. Hence, with this equipment $n_{\mathrm{MAX}}=d \times 255$, where $d$ is a positive integer defining the time window amplitude during the monitoring. Therefore, the $b$-value of Eq. (2) takes the particular form:

$b=\frac{1}{2}\left[\log _{10}(d 255)-\log _{10} n_{0}\right]$,

where in the specific case $n_{0} \neq 0$ is the number of ring-downs counted during a time window of $d \times 120 \mathrm{~s}$.

The above described " $b$-value" analysis has been carried out considering the time series, reported in Table 1, of $\mathrm{AE}$ data collected during the monitoring of damaged zones of Sineo Tower (Fig. 4). The results are depicted in the graph of Fig. 7a. It can be observed that $b$-values in the range $(1.5,2.5)$, far from the critical value $b_{\text {crit }}=1$, may be interpreted as sign of stable damage evolution.

There is a remarkable similarity between the average value $b=1.93$ describing the intensity of AE activity on the tower and the $b$-value measuring the regional level of seismic activity, which is $b=1.85$. Removing earthquakes lower than $M_{L}=2.5$, a good agreement with the linear trend is found (see Fig. 7b), this skimming is consistent with the observational evidence that small earthquakes behave like background noise (Rundle et al., 2003). The intriguing observation on the similarity of the two $b$-values suggests that the $b$-value could be considered as an universal exponent characterizing both the earthquakes distribution and the AE activity monitored on the urban system.

\section{Efforts at earthquake forecasting}

\subsection{Clues for recognizing impending earthquakes}

In this section, we examine a possible application of AE technique in view of probabilistic prediction of the time and place of earthquakes.

Most earthquakes have precursors, which are short term and long-term changes in the Earth that take place prior to an earthquake. Which are the precursors of impending earthquakes? A multitude have been suggested, but it is still not clear which are reliable. Certainly any operative scheme of practical prediction must be based on a combination of clues. In recent years, the major earthquake-prediction effort has been more precise measurements of fluctuations in physical parameters in crustal rocks of seismically active continental areas and suspicious gaps in the regular occurrence of earthquakes in both space and time. More precisely, variation in the seismicity rate is a long-range predictor. In brief, a strong change in the normal background earthquake occurrence is noted. A region in an area of seismic activity that has had little earthquake activity for a number of years is called seismic gap. Seismic gaps are considered to be potential sites for major earthquakes. On the other hand, the increasing pressure in subsurface rocks in the region of the epicentre produce numerous cracks prior to failure and, then, cause changes in rock properties: cracks development in the crustal rocks 

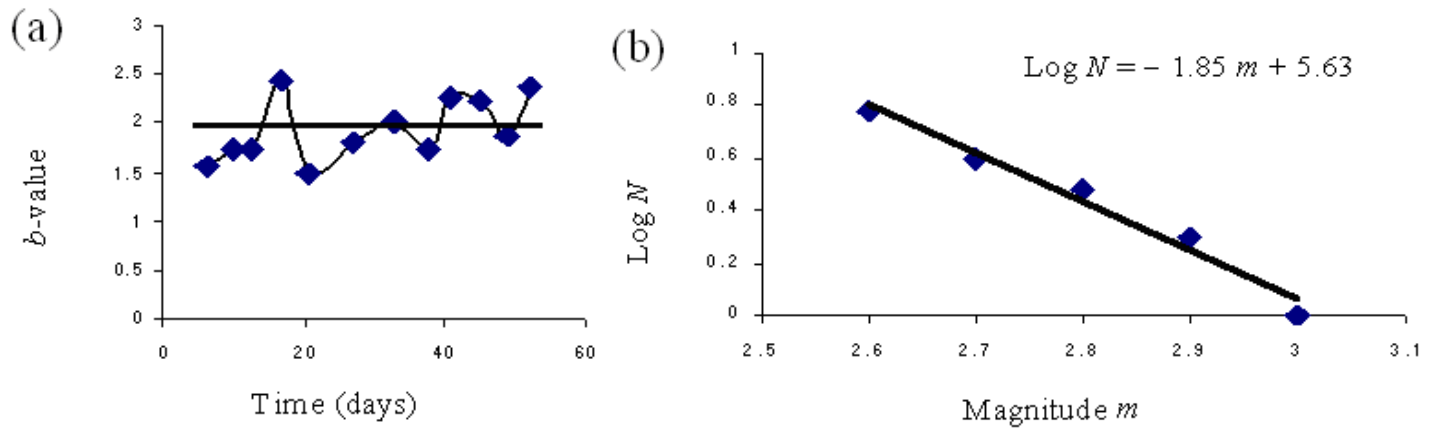

Fig. 7. $b$-value trend of the monitored cracks on Sineo Tower. The straight line represents the average value $b=1.93$ (a). Cumulative number $N$ of earthquakes, with magnitude $\geq m$ as a function of $m$, occurred in Piedmont during the AE monitoring period. The $b$-value is $b=1.85$ (b).

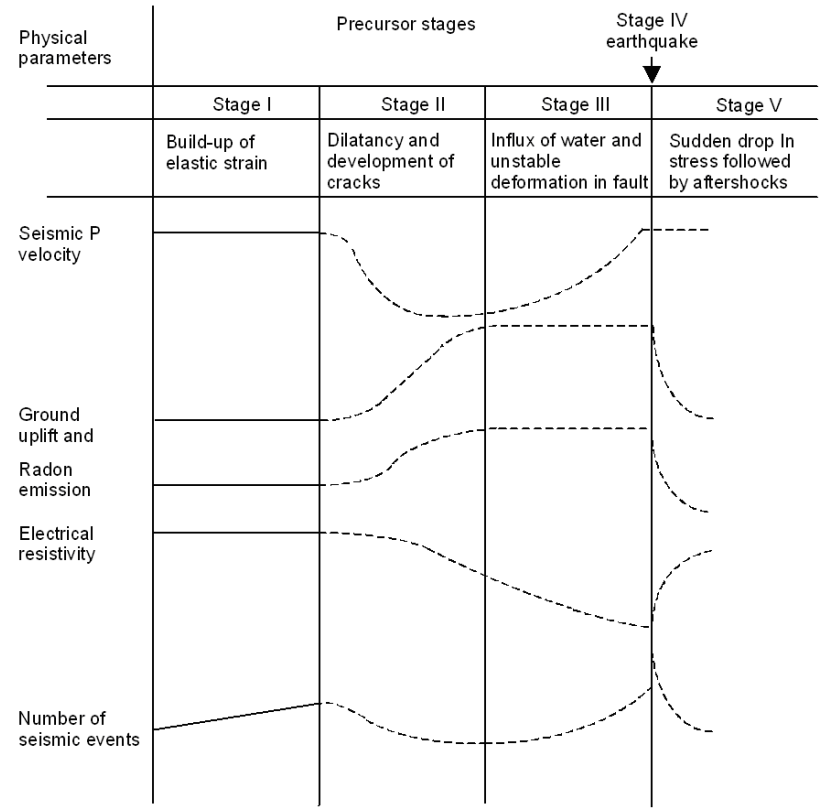

Fig. 8. Physical clues for earthquake prediction (reprinted from Bolt, 1978).

causes the rock volume to swell or dilate. Therefore, velocity drop of seismic waves (of micro-earthquakes) caused by rocks dilatancy is a significant earthquake precursor in an area of seismic activity (Bolt, 1978).

Other precursors due to rock dilatancy and cracks opening are the crustal tilt and elevation changes, decrease of the electrical resistivity of the rocks, and release of radon gas into the atmosphere, which requires very small pores to propagate. As damage process develops, water diffuses from the surrounding rocks into pores and microcracks of increasingly larger size, which meanwhile are forming. As water fills the cracks, the speed of seismic waves through the region begins to increase again, the uplift of the ground ceases, emission of radon from the fresh crack tapers off, and the electrical resistivity decreases further. The subsequent stage is the onset of the earthquake, which is immediately followed by numerous aftershocks occurring in the area. A synthetic representation of these fluctuations is given in Fig. 8 (Bolt, 1978).

\subsection{From acoustic emission to earthquakes}

When dealing with cracks of very small linear size, the mechanically vibrating structures shall be correspondingly very short, and the AE shall be in the ultrasonic range. This is due to the principle of the pendulum, whose frequency $f$ is in inverse relation to its length $l: f^{-1}=2 \pi \sqrt{l / g}$. As soon as the size of the crack increases, either due to the enlargement of one previous crack, or to the coalescence of several previous smaller cracks (or both), the AE shall correspondingly become of progressively lower frequency, until eventually leaving the ultrasonic range and reaching the sonic range, which is the well-known seismic roar. Hence, $\mathrm{AE}$ techniques have the potential for effectively monitoring the stress propagation through the crust and, in effect, some Italian researchers have collected continuous AE records during several years on the Gran Sasso massif, in central Apennines. In effect, an AE paroxysm, i.e. a large and almost abrupt increase of the AE signal, was observed $\sim 400 \mathrm{~km}$ far from the epicentral area several months before the eventual occurrence of the Assisi earthquake (Gregori and Paparo, 2004; Gregori et al., 2005). The progressively lower frequency (from $\sim 200 \mathrm{kHz}$ to $\sim 25 \mathrm{kHz}$ ) of detected AE as time elapsed appeared consistent with the expectation that high-frequency AE, i.e. $\sim 200 \mathrm{kHz}$, is very likely to be associated with the yield of small cracks within the crust, which later coalesce into flaws of increasingly larger size, which release AE of progressively lower frequency.

In order to correlate AE paroxysms to the previously mentioned seismic precursors, indication that Radon exhalation occurs almost at the same time of the AE paroxysm has been found (Gregori and Paparo, 2004), i.e. 7-8 months before 
the earthquake. The importance of Radon as a seismic precursor is due to the fact that it propagates through some comparatively much smaller cracks associated with $\mathrm{AE}$, unlike heavier gases that require comparatively larger cracks associated with the late low-frequency $\mathrm{AE}(\sim 25 \mathrm{kHz})$, which precedes by few weeks the earthquake.

The time sequence of progressively decreasing bulk of the frequency of the released $\mathrm{AE}$ is represented, as a first approximation, in terms of Dirac $\delta$-functions and, in a more detailed way, in terms of some tail feature, see Fig. 9. Therefore, the potential of the $\mathrm{AE}$ monitoring for earthquake prediction appears promising, since precursor AE signals in the ultrasonic range can be captured well in advance.

In the following, a method to correlate peaks of $\mathrm{AE}$ activity in masonry of two medieval towers rising in the centre of Alba to regional seismicity is proposed. The underlying assumption is that the oscillations of the ground propagate through the towers, which behaved as sensitive earthquake receptors. The adopted PZT transducers, sensitive in the range from 50 to $500 \mathrm{kHz}$, when applied to the surface of the structural elements of the towers appear thus capable of capturing the $\mathrm{AE}$ in the ultrasonic range rising from the ground.

4.3 Correlation between $\mathrm{AE}$ activity in the towers and regional seismicity

A definitive method to assess any connection between $\mathrm{AE}$ events and earthquakes separated in space and time probably does not exist. Along the lines of studies on space-time correlation between earthquakes, here we propose a method of statistical data analysis for calculating the degree of correlation both in space and in time between a time series of $\mathrm{AE}$ records and the local seismic records collected in the same time period. The analysis is based on the space-time correlation combined generalisation of the well-known GrassbergerProcaccia correlation integral (Grassberger and Procaccia, 1983), which leads to a visualisation of both spatial and temporal correlations. The space-time combined correlation integral has been defined as follows:

$$
\begin{aligned}
C(r, \tau) \equiv & \frac{1}{N_{E Q} N_{A E}} \sum_{k=1}^{N_{E Q}} \sum_{j=1}^{N_{A E}} \Theta\left(r-\left|\boldsymbol{x}_{k}-\boldsymbol{x}_{j}\right|\right) \\
& \cdot\left(\tau-\left|t_{k}-t_{j}\right|\right)
\end{aligned}
$$

where $N_{A E}$ is the number of the AE paroxysms recorded on the Sineo Tower from 16 September 2000 to 7 November $2000, N_{E Q}$ is the number of earthquakes in the surrounding area recorded during the same period (see Table 1), and $\Theta$ is the Heaviside step function $(\Theta(x)=0$ if $x \leq 0$ and $\Theta(x)=1$ if $x>0)$. The $k$-index runs over all the recorded seismic events $\left\{\boldsymbol{x}_{k}, t_{k}\right\}$, while $j$ runs over all the recorded AE events $\left\{\boldsymbol{x}_{j}, t_{j}\right\}$. Therefore, among all the possible pairs made taking an AE event and a seismic event, the sum in Eq. (4) counts those whose mutual epicentral distance is $\left|\boldsymbol{x}_{k}-\boldsymbol{x}_{j}\right| \leq r$ and
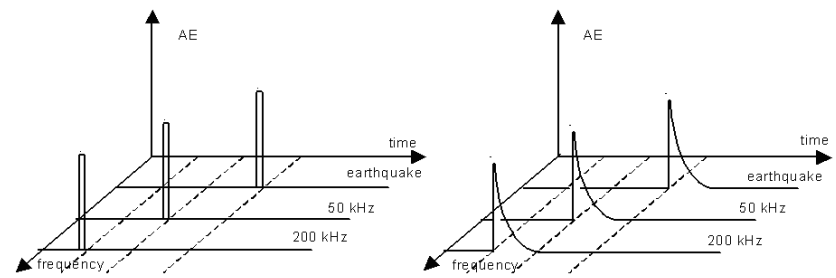

Fig. 9. The bulk of the frequency of the released AE decreases vs. time, and, as a first-order approximation, the phenomenon can be depicted in terms of Dirac $\delta$-functions (a). Upon closer physical consideration, every $\delta$-function should be substituted by a lognormal distribution (b) (Reprint from Gregori and Paparo, 2004).

whose mutual time distance is $\left|t_{k}-t_{j}\right| \leq \tau$ (see Table 1 and Table 2). Therefore, $C(r, \tau)$ is the occurrence probability of two events, one earthquake and one AE event, whose spatial distance is less than $r$ and time distance is less than $\tau$. Without further hypotheses, Eq. (4) permits to assess the degree of correlation without specifying whether AE events play as the precursor or the effect towards earthquakes. In fact, the condition $\left|t_{k}-t_{j}\right| \leq \tau$ does not consider the chronological order between the two events.

Since time series of $\mathrm{AE}$ and earthquakes are two sets strictly interwoven in the time domain, the question whether a given $\mathrm{AE}$ paroxysm occurring in the towers acts as a seismic precursor or is due to possible developing microdamages subsequent to an earthquake is open. Here we propose a probabilistic answer, based on the available data, which can be found considering AE events once as precursors and next as aftershocks of earthquakes, and then comparing the obtained conditioned probability distributions in order to discover the prevailing trend. This analysis has been performed using the modified correlation integral:

$$
\begin{aligned}
C_{ \pm}(r, \tau) \equiv & \frac{1}{N_{E Q} N_{A E}} \sum_{k=1}^{N_{E Q}} \sum_{j=1}^{N_{A E}} \Theta\left(r-\left|\boldsymbol{x}_{k}-\boldsymbol{x}_{j}\right|\right) \\
& \cdot \Theta\left(\tau-\left|t_{k}-t_{j}\right|\right) \cdot \Theta\left( \pm\left(t_{k}-t_{j}\right)\right),
\end{aligned}
$$

where signs "plus" or "minus" in the last $\Theta$-function are used to account $\mathrm{AE}$ events respectively as seismic precursors or aftershocks.

For example, the function $C_{+}(r, \tau)$ gives the probability that a peak of $\mathrm{AE}$, detected at a certain time, will be followed by an earthquake in the following $\tau$ days and within a radius of $r$ kilometres from the AE monitoring site. Varying the thresholds $r$ and $\tau$ in Eqs. (5), two cumulative probability distributions have been constructed, one for each adopted prescription (sign "plus" or "minus") and then the corresponding conditioned probability density functions have been derived and represented in Figs. 10 and 11. It results that AE activity on the tower was more highly correlated to the seismic activity occurred within a radius of $\sim 40 \mathrm{~km}$. A certain diversity comes out examining time correlation, since 

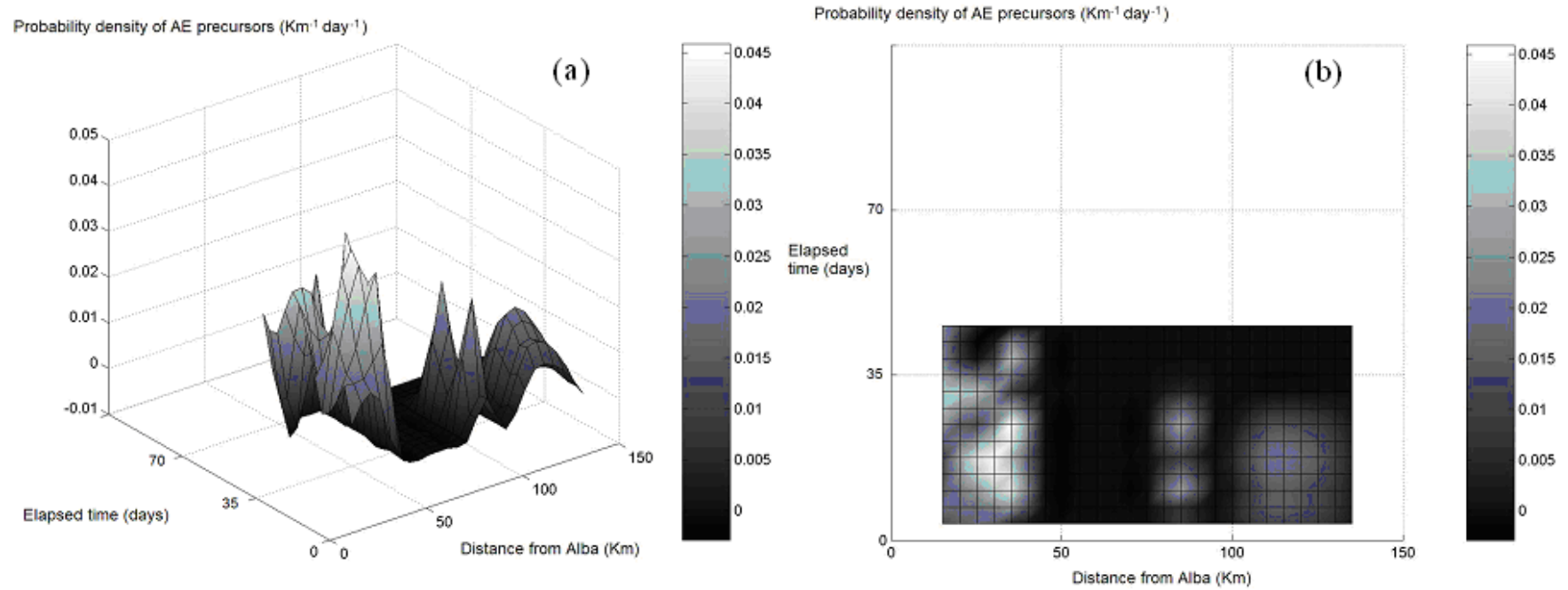

Fig. 10. Probability density that an earthquake and its AE precursor on Sineo Tower occur, as a function of their mutual space and time separations: 3-D representation (a), and grayscale colour map (b).

Probability density of $\mathrm{A}$ E damage aftershocks ( $\mathrm{Km}^{-1}$ day $\left.{ }^{-1}\right)$

Probability density of $\mathrm{AE}$ damage aftershocks $\left(\mathrm{Km}^{-1}\right.$ day $\left.\mathrm{y}^{\prime}\right)$

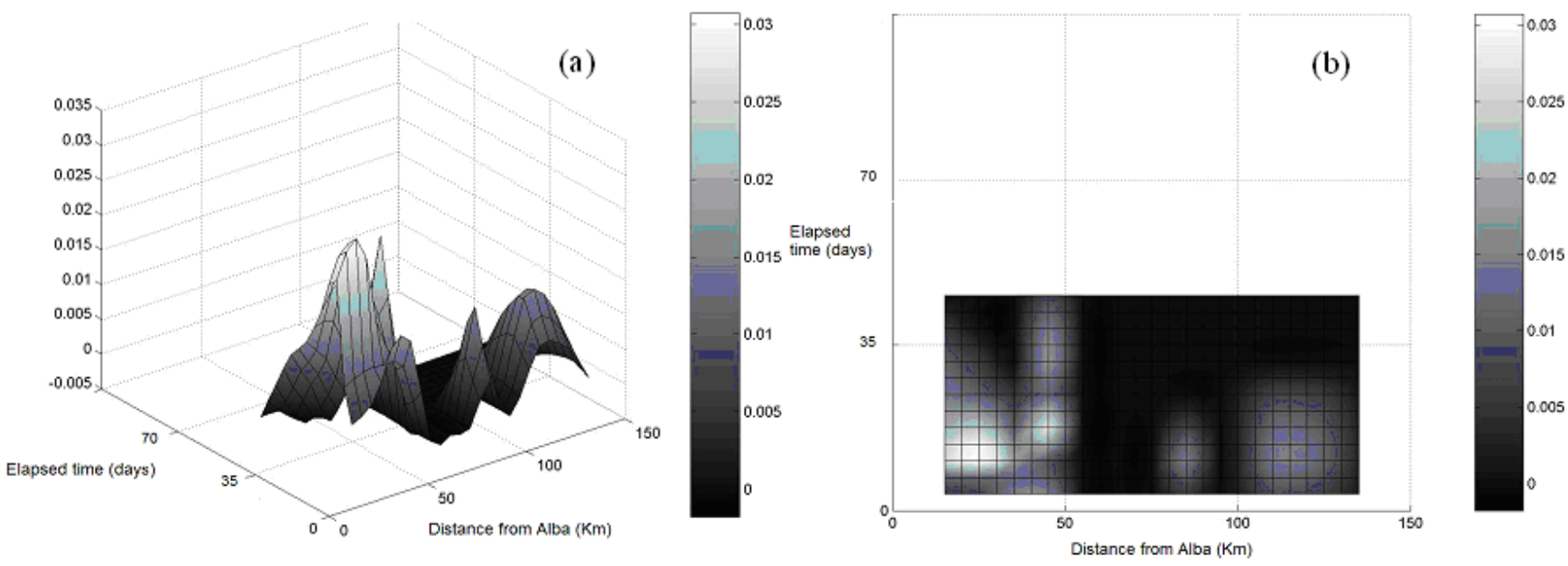

Fig. 11. Probability density that an earthquake and its AE damage-aftershocks on Sineo Tower occur, as a function of their mutual space and time separations: 3-D representation (a), and grayscale colour map (b).

AE precursors appear more long-term correlated to earthquakes (within $\sim 35$ days) than $\mathrm{AE}$ aftershocks (within $\sim 15$ days).

\subsection{Fractal dimensions from space-time correlation integral}

Spatial clustering of earthquakes around the $\mathrm{AE}$ monitoring site and their time coupling to AE events can also be studied through the fractal geometry. From the space-time combined correlation integral of Eq. (4) the time correlation dimension $D_{t}$ and the space correlation dimension $D_{s}$ can be defined respectively as (Tosi et al., 2004):

$C(r, \tau) \sim \tau^{D_{t}}$, and:

$C(r, \tau) \sim r^{D_{s}}$.

By varying the value for $\tau$, a plot can be made to represent the changing value of the correlation integral $C(r, \tau)$ versus the spanned time window $\tau$. Therefore, $D_{t}$ is the slope of $C(r, \tau)$ vs. $\tau$ on the $\log -\log$ plot. Similarly, $D_{s}$ can be obtained by plotting the correlation integral versus the distance $r$. The behaviour of the fractal exponents $D_{t}$ and $D_{s}$ as functions of $r$ and $\tau$ characterize the clustering features of hypocentres around the $\mathrm{AE}$ monitoring site and the time coupling of seismic events to AE events. It can be noted that each seismic event is marked in this section not by its 

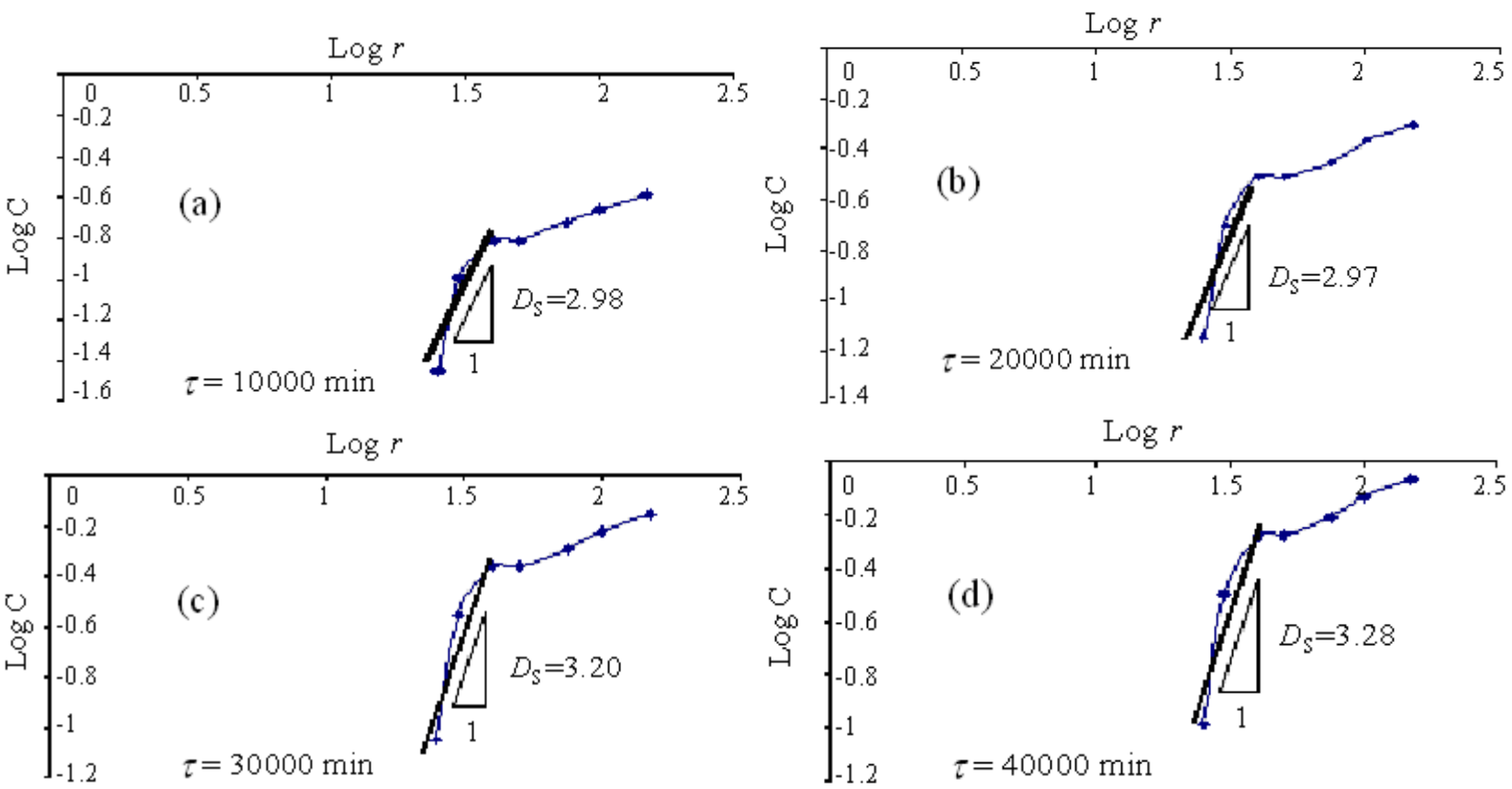

Fig. 12. Fractal dimensions of spatial distribution of the earthquakes hypocenters around the AE monitoring site obtained from the correlation integral (Eq. 4).Different time windows are considered from $\tau=10000 \mathrm{~min}$, panel (a), to $\tau=40000 \mathrm{~min}$, panel (d).

epicentre but rather by its hypocentre; in this way, as shown below, a consistent interpretation for $D_{s}$ will be found.

High values of $D_{s}$ represent a clustered spatial distribution of the hypocentres, while high values of $D_{t}$ reflect a causal connection between earthquakes and $\mathrm{AE}$ events.

On the contrary, low values of $D_{t}$ between $\tau_{1}$ and $\tau_{2}$, for example, describe a slow increase of the cumulative distribution $C(r, \tau)$. Therefore, the occurrence probability of two events, one seismic and the other acoustic, is small if their time distance is included in the range $\left(\tau_{1} \tau_{2}\right)$. In this case, namely, earthquakes and AE events are weakly correlated.

Fractal dimension $D_{s} \cong 3$, representing earthquake hypocentres clustered in a Earth's volume around the $\mathrm{AE}$ monitoring site, is found for different values of $\tau$ (Fig. 12). The radius of the cluster is defined by the switch point, beyond which the rapid increase of correlation integral of the form $C(r, \tau) \sim r^{3}$ turns into a gentler power-law increase, hallmark of a more rarefied spatial distribution of the hypocentres. Considering different values of the spanned time window $\tau$ a common value of $r_{i} \sim 40 \mathrm{~km}$ found, for the radius of the clusters, shows that bursts of AE activity are correlated to earthquakes which occur within a radius $r_{i}$ of about $40 \mathrm{~km}$. Since $C(r, \tau)$ counts AE events irrespective if they are seismic foreshocks or aftershocks, the observed evidence suggests that at the considered magnitudes, within $r_{i}$ and with $\tau$ ranging from 10000 up to $40000 \mathrm{~min}$, lie the most hazardous earthquakes for which the towers are more sensitive receptors.
Studying the time coupling of AE activity on the Sineo Tower to regional seismicity with the fractal $D_{t}$ approach, AE precursors result to be more long-term correlated than AE damage-aftershocks (see Fig. 13). In fact, considering AE events as seismic precursors, high values of $D_{t}$ are found for large time intervals, while low values result for short time intervals. The opposite occurs considering AE events as damage-aftershocks. In this sense, $\mathrm{AE}$ on structures seems to be both a good long-term seismic precursor and meanwhile a reliable damage index.

\section{Conclusions}

Besides the canonical use in non-destructive tests, the heuristic potential of AE monitoring of civil structures for environmental sciences (such as earthquake prediction) appears very intriguing. A method of correlating AE activity on a medieval masonry tower subjected to long-term monitoring to regional seismicity has been proposed. From the evidence in the case study, similar contributions to AE activity in the tower result from inner sources (structural damage) and external sources (crustal damage). Two qualitatively very similar phenomena such as acoustic emission and earthquakes become, when mutually correlated, two aspects of a unique phenomenon which looks the same on any scale or selfsimilar. That is reflected by the common $b$-value shared by these two classes of phenomena. 

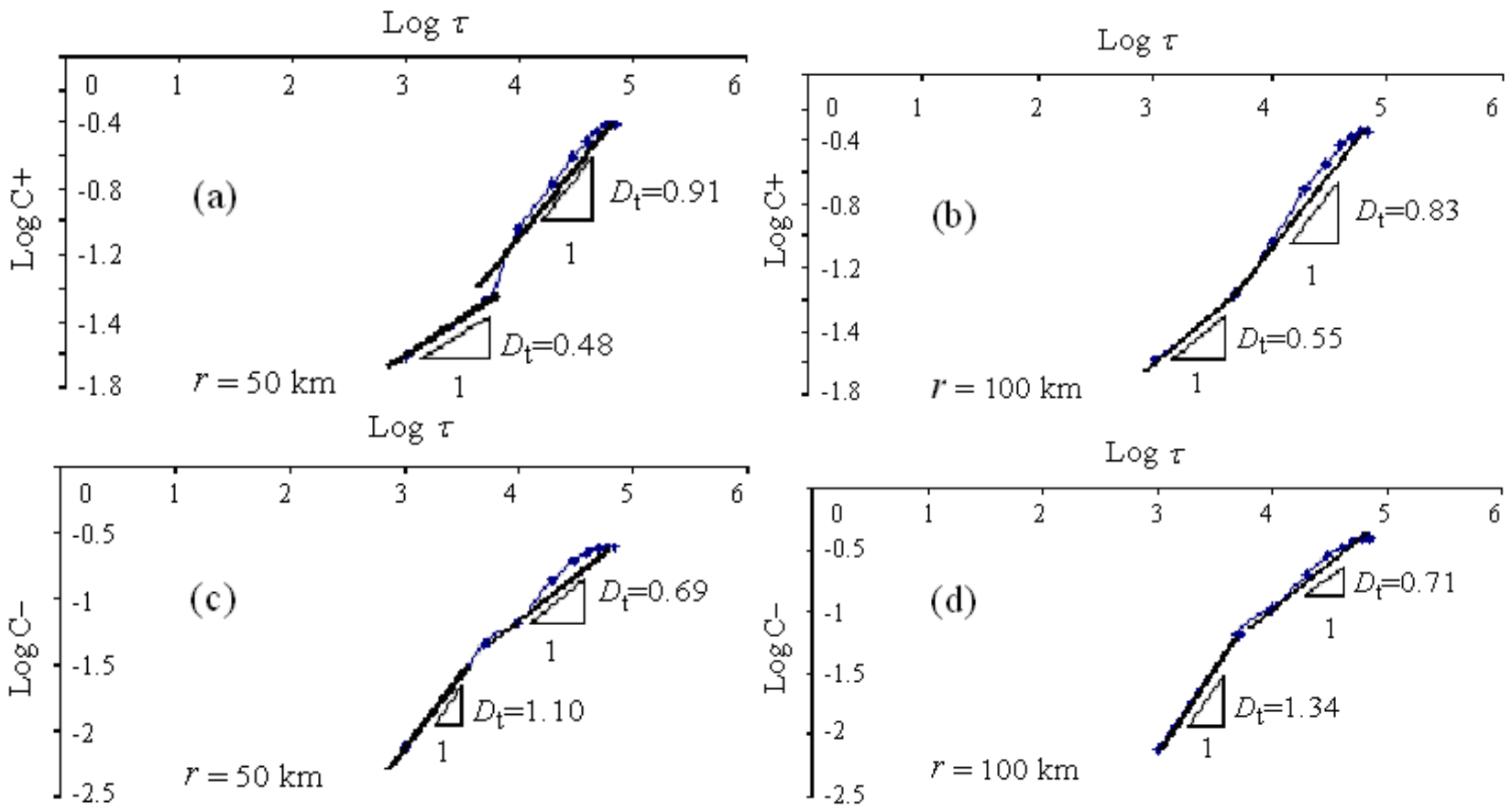

Fig. 13. Fractal dimensions of time correlation between earthquakes and AE events on the monitored site obtained from the correlation integral (Eq. 5). In the panels (a) and (b), the correlation integrals versus time interval related to AE precursors are represented, for a spanned region size respectively of 50 and $100 \mathrm{~km}$ : high values of $D_{t}$, reflecting strong correlation, are observed for long intervals elapsing between AE precursors and earthquakes. In the panels (c) and (d), the correlation integrals versus time interval related to AE aftershocks are represented, for a spanned region size respectively of 50 and $100 \mathrm{~km}$ : high values of $D_{t}$ are observed for short intervals elapsing between earthquakes and $\mathrm{AE}$ aftershocks.

Furthermore, in this work by applying the GrassbergerProcaccia correlation algorithm and its interpretation by fractal geometry in the aim of explaining the connection between regional seismicity and acoustic emission emerging from specific structures, it has been proved that these last are good sensitive receptors for earthquakes occurring within a radius of $40 \mathrm{~km}$ and for waiting times comprised between 10000 and $40000 \mathrm{~min}$.

In this sense, true progress can be realistically achieved by means of the simultaneous operation of suitable arrays of $\mathrm{AE}$ monitoring sites, adequately placed in the territory, e.g. in the order of $\sim 1000$ over one large regional area.

Acknowledgements. This research was carried out with the financial support of the Ministry of University and Scientific Research (MIUR) and of the European Union (EU). The authors would like to thank the City of Alba and M. Reggio for their indispensable collaboration and Architects M. Aprile and L. Bacco for the technical support provided in the structural monitoring.

Edited by: M. Contadakis

Reviewed by: K. Eftaxias

\section{References}

Anzani, A., Binda, L., and Mirabella Roberti, G.: The effect of heavy persistent actions into the behaviour of ancient masonry, Materials and Structures, 33, 251-261, 2000.

Bolt, B. A.: Earthquakes, W. H. Freeman and Company, S. Francisco and London, 1978.

Brindley, B. J., Holt, J., and Palmer, I. G.: Acoustic Emission-3: the use of ring-down counting, Non-Destructive Testing, 6, 299-306, 1973.

Carpinteri, A.: Scaling laws and renormalization groups for strength and toughness of disordered materials, Int. J. Solids and Structures, 31, 291-302, 1994.

Carpinteri, A. and Bocca, P.: Damage and diagnosis of materials and structures, Proc. of DDMS 91, Politecnico di Torino, Pitagora, Bologna, 1991.

Carpinteri, A., Invernizzi, S., and Lacidogna, G.: In situ damage assessment and nonlinear modeling of an historical masonry tower, Engineering Structures, 27, 387-395, 2005.

Carpinteri, A. and Lacidogna, G.: Damage monitoring of an historical masonry building by the acoustic emission technique, Materials and Structures, 39, 161-167, 2006a.

Carpinteri, A. and Lacidogna, G.: Structural monitoring and integrity assessment of medieval towers, ASCE J. Structural Engineering, 32, 1681-1690, 2006b.

Carpinteri, A., Lacidogna, G., and Niccolini, G.: Critical behaviour 
in concrete structures and damage localization by acoustic emission, Key Engineering Materials, 312, 305-310, 2006.

Carpinteri, A., Lacidogna, G., and Pugno, N.: A fractal approach for damage detection in concrete and masonry structures by acoustic emission technique, Acoustique et Techniques, 38, 3137, 2004.

Carpinteri, A., Lacidogna, G., and Pugno, N.: Structural damage diagnosis and life-time assessment by acoustic emission monitoring, Engineering Fracture Mechanics, 74, 273-289, 2007.

Colombo, S., Main, I. G., and Forde, M. C.: Assessing damage of reinforced concrete beam using " $b$-value" analysis of acoustic emission signals, J. Materials in Civil Engineering ASCE, 15, 280-286, 2003.

Grassberger, P. and Procaccia, I.: Characterization of strange attractors, Phys. Rev. Lett., 50, 346-349, 1983.

Gregori, G. P. and Paparo, G.: Acoustic emission (AE). A diagnostic tool for environmental sciences and for non destructive tests (with a potential application to gravitational antennas), Meteorological and geophysical fluid dynamics, edited by: Schroeder, W., Science Edition, Bremen, 166-204, 2004.
Gregori, G. P., Paparo, G., Poscolieri, M., and Zanini, A.: Acoustic emission and released seismic energy, Nat. Hazards Earth Syst. Sci., 5, 777-782, 2005, http://www.nat-hazards-earth-syst-sci.net/5/777/2005/.

Pollock, A. A.: Acoustic Emission-2: acoustic emission amplitudes, Non-Destructive Testing, 6, 264-269, 1973.

Richter, C. F.: Elementary seismology, W. H. Freeman and Company, San Francisco and London, 1958.

Rundle, J. B., Turcotte, D. L., Shcherbakov, R., Klein, W., and Sammis, C.: Statistical physics approach to understanding the multiscale dynamics of earthquake fault systems, Rev. Geophys., 41, 1019, 2003.

Tosi, P., De Rubeis, V., Loreto, V., and Pietronero, L.: Space-time combined correlation integral and earthquake interactions, Ann. Geophys., 47, 1849-1854, 2004, http://www.ann-geophys.net/47/1849/2004/.

Zapperi, S., Vespignani, A., and Stanley, H. E.: Plasticity and avalanche behaviour in microfracturing phenomena, Nature, 388 , 658-660, 1997. 